\title{
Observer design for two-wheeled vehicle : A Takagi-Sugeno approach with unmeasurable premise variables
}

\author{
Dalil Ichalal, Hichem Arioui, and Said Mammar
}

\begin{abstract}
This paper is dedicated to the problem of observer design for Takagi-Sugeno (T-S) nonlinear systems with unmeasurable premise variables (TSUPV) and application to autonomous bicycle system. The main idea is based on the use of differential mean value theorem combined to the sector nonlinearity transformation. The objective of this approach is to make the state estimation error dynamic on a T-S form which allows to apply the classical Lyapunov analysis to derive convergence conditions. The design algorithm is proposed in terms of linear matrix inequalities (LMI). To illustrate the proposed methodology, a nonlinear bicycle model is considered.

Index Terms-Nonlinear systems, Takagi-Sugeno nonlinear systems, LMI, observer design, two-wheeled vehicles
\end{abstract}

\section{INTRODUCTION}

State estimation and observer design of nonlinear systems is an important problem in modern control. Early work on this problem dates back to the work of Thau [27] where the author proposed an extension of the Luenberger observer [14] to Lipschitz systems. Sufficient conditions are then obtained for the convergence of the state estimation error toward zero but no methodology has been proposed for designing the observer. Thereafter, in [20], an iterative approach is proposed for observer gain design, however, the algorithm may fail even if the system is observable. In [21], Rajamani obtained necessary and sufficient conditions on the observer matrix that ensure asymptotic stability of the state estimation error and proposed a design procedure, based on the gradient optimization method. He discussed also the equivalence between the stability condition and the $H_{\infty}$ minimization in the standard form, and pointed out that this design method was not solvable since the regularity assumptions are not satisfied. Recently, in [19], the result of Rajamani [18] is extended, the authors proposed a dynamic observer and provide a solution to the problem of regularity assumptions by modifying the $H_{\infty}$ problem. Other classes of nonlinear systems are also studied in the literature to design observers for nonlinear systems, namely Linear Parameter Varying systems (LPV) [3] and bilinear systems.

Recently, an extensive studies are made on a new structure which was introduced in [24]. It is based on the decomposition of the operating space of the system in some zones and each zone is represented by a linear model. By defining adequate nonlinear functions satisfying the convex sum property, the behavior of the nonlinear system is modeled in a compact set of the state space. Obtaining a T-S model

The authors are with the Laboratory of Informatique, Biologie Intégrative et Systèmes Complexes (IBISC), Université d'Evry Val d'Essonne, 40, rue du Pelvoux, 91020 Courcouronnes, \{Dalil.Ichalal, Hichem.Arioui, Said.Mammar\}eiup.univ-evry.fr can be performed by linearization of a nonlinear model around some operating points. It can also be obtained by black-box approaches allowing to identify the parameters of the model from input-output data. Finally, interesting mathematical transformations known under the sector nonlinearity transformations can also provide a T-S model [25], [16]. Indeed, this transformation allows to obtain an exact $\mathrm{T}-\mathrm{S}$ representation of a general nonlinear model with no information loss, in a compact state space.

Takagi-Sugeno model has proved its effectiveness in the analysis, control and observation of nonlinear systems. Indeed, it gives a simpler formulation from the mathematical point of view to represent the behavior of nonlinear systems [24]. Thanks to the convex sum property of the weighting functions, it is possible to generalize some tools developed in the linear domain to nonlinear systems. This representation is very interesting in the sense that it simplifies the stability study of nonlinear systems and controller/observer design. In [6], [11], [12], the stability and stabilization tools are proposed. In [2], [15], the problem of state estimation and diagnosis of T-S systems is addressed. The proposed approaches rely on the generalization of the classical observers (Luenberger Observer [14] and Unknown Input Observer (UIO) [8]) for nonlinear domain. The major problem of these results is the conservatism of the conditions which is difficult to solve. Some works are dedicated to reduce this conservatism of the stability condition. For example, in [22], the Polya's theorem is used in order to reduce the conservatism related to the negativity of a sum matrices inequalities. In [13], the authors proposed a new approach for discrete time T-S systems, it is based on the evaluation of the difference of Lyapunov function between $m>1$ samples.

Let us consider a nonlinear system described by a T-S model

$$
\dot{x}(t)=\sum_{i=1}^{r} \mu_{i}(\xi(t))\left(A_{i} x(t)+B_{i} u(t)\right), \quad y(t)=C x(t)
$$

where $x(t) \in \mathbb{R}^{n}$ is the state vector, $u(t) \in \mathbb{R}^{m}$ is the input vector, and $y(t) \in \mathbb{R}^{p}$ represents the output vector. $A_{i} \in \mathbb{R}^{n \times n}$, $B_{i} \in \mathbb{R}^{n \times m}, C \in \mathbb{R}^{p \times n}$ are known matrices. The functions $\mu_{i}(\xi(t))$ are the weighting functions depending on the variables $\xi(t)$ which can be measurable (as the input or the output of the system) or non measurable variables (as the state of the system). These functions verify the following properties

$$
\sum_{i=1}^{r} \mu_{i}(\xi(t))=1, \quad 0 \leq \mu_{i}(\xi(t)) \leq 1 \quad \forall i \in\{1, \ldots, r\}
$$


In this work the considered premise variable $\xi(t)$ depends on the state of the system which is unmeasurable.

The problem of state estimation of nonlinear systems using T-S model approach has been addressed with different methods, the most of the published works considered T-S models with measurable premise variables [1], [18], [15], [2]. It is clear that the choice of measurable premise variables offers a good simplicity to generalize the methods already developed for linear systems. But in the case where the premise variables are not measurable, the problem becomes harder. However, this formalism is very important in both the exact representation of the nonlinear behavior by T-S model (see the simulation example) and in diagnosis method based on observer banks to detect and isolate actuator and sensor faults. Indeed in this case, the use of measurable premise variables requires to develop two different models, the first using the input $u(t)$ in the premise variable to detect and isolate sensor faults, and the second using the output of the system for actuator faults. T-S model with unmeasurable premise variables allows to develop only one model of the system behavior to detect and isolate actuator and sensor faults using observer banks. Furthermore, the T-S models with unmeasurable premise variables may represent a larger class of nonlinear systems compared to the T-S model with measurable premise variables [28]. In the literature, a few works are devoted to the case of unmeasurable decision variables, nevertheless, we can cite [5], [17], [4] where the authors proposed the fuzzy Thau-Luenberger observer which is an extension of the classical Luenberger observer and in [28], a filter estimating the state and minimizing the effect of disturbances is proposed.

In this paper a new method is proposed for state estimation of nonlinear systems. It is based on the use of the TakagiSugeno model representing the behavior of the nonlinear system. The contribution of this work concerns the consideration of the case when the premise variables of the T-S model are not measurable (as the state of the system), this situation is commonly encountered when using the sector nonlinearity approach [25]. The main results on observer design are given in sections II. In section III, an application of the observer is proposed on a nonlinear autonomous bicycle. In this application, a first observer is proposed which is designed by the proposed methods. Secondly, an other observer is designed to enhance the performances, especially to reduce the oscillatory phenomenon in the transient phase of the convergence of the observer. This task is performed by a pole assignment in a chosen LMI region.

\section{OBSERVER DESIGN}

Let consider the T-S system

$$
\dot{x}(t)=\sum_{i=1}^{r} \mu_{i}(x(t))\left(A_{i} x(t)+B_{i} u(t)\right), \quad y(t)=C x(t)
$$

Using the following notations

$$
X_{\mu}=\sum_{i=1}^{r} \mu_{i}(x(t)) X_{i}
$$

where $X_{i}$ stands for $A_{i}$ or $B_{i}$. The system (3) becomes

$$
\left\{\begin{aligned}
\dot{x}(t) & =A_{\mu} x(t)+B_{\mu} u(t) \\
y(t) & =C x(t)
\end{aligned}\right.
$$

The observer is given by

$$
\left\{\begin{array}{l}
\dot{\hat{x}}(t)=A_{\hat{\mu}} \hat{x}(t)+B_{\hat{\mu}} u(t)+L_{\hat{\mu}}(y(t)-\hat{y}(t)) \\
\hat{y}(t)=C \hat{x}(t)
\end{array}\right.
$$

The state estimation error $e(t)=x(t)-\hat{x}(t)$ is governed by the following differential equation

$$
\dot{e}(t)=f(x, u)-f(\hat{x}, u)-L_{\hat{\mu}} C e(t)
$$

where $f(x, u)=A_{\mu} x(t)+B_{\mu} u(t)$ and $f(\hat{x}, u)=A_{\hat{\mu}} \hat{x}(t)+$ $B_{\hat{\mu}} u(t)$. The function $f(\hat{x}, u): \mathbb{R}^{n} \rightarrow \mathbb{R}^{n}$ is assumed to be Lipschitz continuous.

Note that the stability analysis of (7) cannot be directly achieved with the help of the tools developed for T-S systems with measurable premise variables. Indeed, the fact that the premise variable is the state of the system leads to a more complex form of the state estimation error (see equation (7)). The key point of the proposed observer design is to obtain a suitable form of the state estimation error in order to re-use the tools proposed for stability and relaxed stability analysis of T-S systems with measurable premise variables. In conclusion, the objective is to find the gain $L_{\mu}$ of the observer (6) that stabilizes (7).

The $n$ different entries of the nonlinear vector function $f(z): \mathbb{R}^{n} \rightarrow \mathbb{R}^{n}$, are denoted $f_{i}(z)$, it follows

$$
f(z)=\left[\begin{array}{lll}
f_{1}(z) & \ldots & f_{n}(z)
\end{array}\right]^{T}
$$

where $f_{i}(z): \mathbb{R}^{n} \rightarrow \mathbb{R}, i=1, \ldots, n$.

Let us denote $e_{s}(i)$ the vector of $\mathbb{R}^{s}$ with all entries being null, except the $i^{t h}$ being equal to 1 as given below

$$
e_{s}(i)=\left(\begin{array}{ccccccc}
0 & \cdots & 0 & 1 & 0 & \cdots & 0 \\
1 & & i-1 & i & i+1 & & s
\end{array}\right)^{T}
$$

The function $f(z)$ can be written as follows

$$
f(z)=\sum_{i=1}^{n} e_{n}(i) f_{i}(z)
$$

Theorem 1: Consider $f_{i}(z): \mathbb{R}^{n} \rightarrow \mathbb{R}$. Let $a, b \in \mathbb{R}^{n}$. If $f_{i}(z)$ is differentiable on $[a, b]$ then there exists a constant vector $z^{i} \in \mathbb{R}^{n}$, satisfying $\left.\xi^{i} \in\right] a, b\left[\right.$ (i.e. $\left.\xi_{j}^{i} \in\right] a_{j}, b_{j}[$, for $j=1, \ldots, n$ ), such that

$$
f_{i}(a)-f_{i}(b)=\frac{\partial f_{i}}{\partial z}\left(\xi^{i}\right)(a-b)
$$

Applying the theorem 1 on (7), it is obtained for $a, b \in \mathbb{R}^{n}$

$$
f(a)-f(b)=\sum_{i=1}^{n} \sum_{j=1}^{n} e_{n}(i) e_{n}^{T}(j) \frac{\partial f_{i}}{\partial z_{j}}\left(\xi^{i}\right)(a-b)
$$

Using (12), the state estimation error (7) can be then transformed into

$$
\dot{e}(t)=\left(\sum_{i=1}^{n} \sum_{j=1}^{n} e_{n}(i) e_{n}^{T}(j) \frac{\partial f_{i}}{\partial x_{j}}\left(\xi^{i}\right)-L_{\hat{\mu}} C\right) e(t)
$$


Assumption 1: Assume that $f\left(z^{i}\right)$ is a differentiable function satisfying, for $i=1, \ldots, n$ and $j=1, \ldots, n$

$$
a_{i j} \leq \frac{\partial f_{i}}{\partial x_{j}}\left(z^{i}\right) \leq b_{i j}
$$

Each nonlinearity $\frac{\partial f_{i}}{\partial x_{j}}\left(z^{i}\right)$ can be represented by

$$
\frac{\partial f_{i}}{\partial x_{j}}\left(z^{i}\right)=\sum_{l=1}^{2} v_{i j}^{l}\left(z^{i}\right) \tilde{a}_{i j l}
$$

where $\tilde{a}_{i j 1}=a_{i j}$ and $\tilde{a}_{i j 2}=b_{i j}$ and

$$
\begin{array}{r}
v_{i j}^{1}\left(\xi^{i}\right)=\frac{\frac{\partial f_{i}}{\partial x_{j}}\left(\xi^{i}\right)-a_{i j}}{b_{i j}-a_{i j}}, \quad v_{i j}^{2}\left(\xi^{i}\right)=\frac{b_{i j}-\frac{\partial f_{i}}{\partial x_{j}}\left(z^{i}\right)}{b_{i j}-a_{i j}} \\
\sum_{l=1}^{2} v_{i j}^{l}\left(\xi^{i}\right)=1, \quad 0 \leq v_{i j}^{l}\left(\xi^{i}\right) \leq 1, \quad l=1,2
\end{array}
$$

Using (13) and (15), the dynamic of the state estimation error is represented by

$$
\dot{e}(t)=\left(\mathscr{A}_{h}-L_{\hat{\mu}} C\right) e(t)
$$

where $\mathscr{A}_{h}=\sum_{i=1}^{q} h_{i}(\xi) \mathscr{A}_{i}$ and $q=2^{n^{2}}$. The weighting function $h_{i}($.$) are defined by following the sector nonlinearity ap-$ proach in T-S fuzzy [25] systems by using the local weighting functions $v_{i j}$ defined above. The stability of the state estimation error (18) is studied by the quadratic Lyapunov function with common matrix

$$
V(e(t))=e^{T}(t) P e(t), \quad P=P^{T}>0
$$

Its derivative with respect to $t$ is

$$
\dot{V}(e(t))=e^{T}(t)\left(A_{h}^{T} P+P A_{h}-C^{T} L_{\hat{\mu}}^{T} P-P L_{\hat{\mu}} C\right) e(t)
$$

The stability of the state estimation error is ensured if the time derivative of the Lyapunov equation (20) is negative definite, which leads to the following time dependent LMIs

$$
\mathscr{A}_{h}^{T} P+P \mathscr{A}_{h}-C^{T} L_{\hat{\mu}}^{T} P-P L_{\hat{\mu}} C<0
$$

The convex sum property of the weighting functions allows to obtain time independent inequality

$$
\mathscr{A}_{i}^{T} P+P \mathscr{A}_{i}-C^{T} L_{j}^{T} P-P L_{j} C<0, i=1, \ldots, q, j=1, \ldots, r
$$

To express the inequality (22) in term of LMI, the change of variables $K_{j}=P L_{j}$ is used and LMI conditions are obtained as follows

$$
\mathscr{A}_{i}^{T} P+P \mathscr{A}_{i}-C^{T} K_{j}^{T}-K_{j} C<0, i=1, \ldots, q, j=1, \ldots, r
$$

\section{Autonomous TWO-WHEELED VEHICLE}

Driver assistance andsafetyare becomingincreasinglycommonin automotive applications to fight against theproblems relative toroadsafety.Indeed, thenumber of roads deathsdecreases since the introduction of safety systemssuchvehicle stabilization systems: Anti-lock Brake Systems (ABS) and Electronic Stability Control (ESC) and have now become almost standard in every passenger car. Other systems, such as airbags, collision avoidance, adaptive cruise control, etc., are available more recently for four wheels vehicles. For single-track ones such as motorcycles, the delay in this field is clear. Furthermore, the applications can be more complex compared to two-tracked vehicles due to the system dynamics, stability/equilibrium, maneuverability, etc. To deploy such systems on motorcycles, one has to know reliably what the current state of the vehicle is. Various sensors can be used (e.g. lateral acceleration, yaw rate, wheel speeds), which are already used for four-wheeled vehicle applications. However, the major difference concerns the roll angle which can exceed $40^{\circ}$ in some limit cases.

Roll angle is the main characteristics of the Motorcycle lateral dynamics. A good control of motorcycle motions requires an accurate assessment of this quantity and for safety applications also the risk of sliding or friction loss need to be considered. Direct measurement of roll angle and tire slip is not available or very expensive as for the lateral speed (corevit sensor). Some previous work [26] and [23] have addressed these challenges based on estimation and/or observation techniques of these dynamic parameters. The success of these methods remains modest and mainly depends on the model's complexity. Indeed, most studies have considered simple models and generally linear. The reality is far from these assumptions and is highly nonlinear

In this section, the proposed observer is applied in order to estimate the states of an autonomous bicycle. The model of the system is given in the first subsection with its T-S representation. The second subsection illustrates the observer design approach and finally, simulation results are given.

\section{A. Modeling}

The presented model (see the figure 1) in this paper is proposed in [10] and [9] where

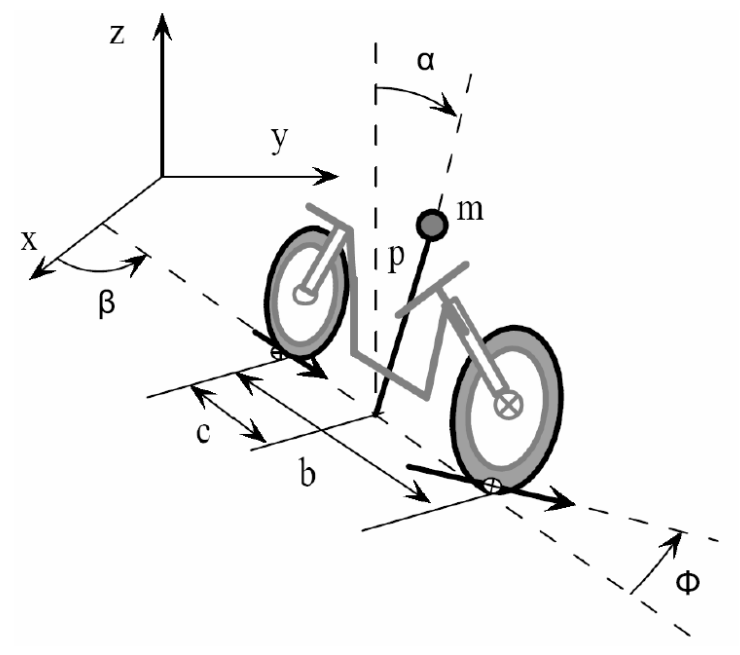

Fig. 1. Bicycle model

- $u(t)(N . m)$, the normalized input torque exerting on the steering angle $\phi$.

- $v(\mathrm{~m} / \mathrm{s})$, the forwarding speed of the motorcycle 
- $p(m)$, the distance from the center of mass to the ground

- $c(m)$, the horizontal distance from the center of mass to the ground contact point of the rear wheel

- $\beta(\mathrm{rad})$, the yaw angle of the motorcycle

- $\alpha(\mathrm{rad})$, the roll angle of the motorcycle

A state space nonlinear model is then obtained as follows

$$
\begin{aligned}
\dot{\beta} & =\psi_{\beta} v \\
\dot{\psi}_{\beta} & =u \\
\dot{\alpha} & =\psi_{\alpha} \\
\dot{\psi}_{\alpha} & =\frac{1}{p}\left(g \sin (\alpha)+\left(1+p \psi_{\beta} \sin (\alpha)\right) \cos (\alpha) \psi_{\beta} v^{2}\right. \\
& +c \cos (\alpha) v u)
\end{aligned}
$$

For simplicity and clearness, the model is re-written in a general state space system where

$$
x(t)=\left(\begin{array}{c}
x_{1}(t) \\
x_{2}(t) \\
x_{3}(t) \\
x_{4}(t)
\end{array}\right)=\left(\begin{array}{c}
\beta \\
\psi_{\beta} \\
\alpha \\
\psi_{\alpha}
\end{array}\right)
$$

The model is then given by

$$
\begin{aligned}
\dot{x}_{1}(t) & =x_{2}(t) v \\
\dot{x}_{2}(t) & =u(t) \\
\dot{x}_{3}(t) & =x_{4}(t) \\
\dot{x}_{4}(t) & =\frac{1}{p}\left(g \sin \left(x_{3}(t)\right)+(1\right. \\
& \left.+p x_{2}(t) \sin \left(x_{3}(t)\right)\right) \cos \left(x_{3}(t)\right) x_{2}(t) v^{2} \\
& \left.+c \cos \left(x_{3}(t)\right) v u\right)
\end{aligned}
$$

which can be also written in a compact form

$$
\dot{x}(t)=f(x, u)
$$

The system is unstable. To stabilize it, the simple control law

$$
u(t)=-k_{1}\left(x_{3}(t)-x_{3 d}(t)\right)-k_{2}\left(x_{4}(t)-x_{4 d}(t)\right)
$$

is proposed, where $x_{3 d}(t)=\sin (t)$ and $x_{4 d}(t)=\cos (t)$ represent the trajectories to track and $k_{1}=k_{2}=10$. The nonlinear trajectories with this control are depicted in the figure 3 .

Note that a T-S model is not unique for a given system. So, in this application, we chose the following model. By using the sector nonlinearity transformation, a T-S model is obtained in the form given in equation (3) with 8 sub models. The chosen premise variables are given by

$$
\left\{\begin{array}{l}
z_{1}(t)=\frac{g}{p} \frac{\sin \left(x_{3}(t)\right)}{x_{3}(t)} \\
z_{2}(t)=\frac{1}{p}\left(1+p x_{2}(t) \sin \left(x_{3}(t)\right)\right) \cos \left(x_{3}(t)\right) v^{2} \\
z_{3}(t)=\frac{c}{p} \cos \left(x_{3}(t)\right) v
\end{array}\right.
$$

and under the assumptions

$$
\left\{\begin{array}{l}
z_{1}^{\min } \leq z_{1}(t) \leq z_{1}^{\max } \\
z_{2}^{\min } \leq z_{2}(t) \leq z_{2}^{\max } \\
z_{3}^{\min } \leq z_{3}(t) \leq z_{3}^{\max }
\end{array}\right.
$$

the local weighting functions are defined by

$$
\begin{array}{ll}
F_{1}^{0}(t)=\frac{z_{1}(t)-z_{1}^{\min }}{z_{1}^{\max }-z_{1}^{\min }}, & F_{1}^{1}(t)=\frac{z_{1}^{\max }-z_{1}(t)}{z_{1}^{\max }-z_{1}^{\min }} \\
F_{2}^{0}(t)=\frac{z_{2}(t)-z_{2}^{\min }}{z_{2}^{\max }-z_{2}^{\min }}, & F_{2}^{1}(t)=\frac{z_{2}^{\max }-z_{2}(t)}{z_{2}^{\max }-z_{2}^{\min }} \\
F_{3}^{0}(t)=\frac{z_{3}(t)-z_{3}^{\min }}{z_{3}^{\max }-z_{3}^{\min }}, & F_{3}^{1}(t)=\frac{z_{3}^{\max }-z_{3}(t)}{z_{3}^{\max }-z_{3}^{\min }}
\end{array}
$$

Finally, the weighting functions of the T-S model are given by

$$
\begin{array}{ll}
\mu_{1}(z(t))=F_{1}^{0}(t) F_{2}^{0}(t) F_{3}^{0}(t), \quad \mu_{2}(z(t))=F_{1}^{0}(t) F_{2}^{0}(t) F_{3}^{1}(t) \\
\mu_{3}(z(t))=F_{1}^{0}(t) F_{2}^{1}(t) F_{3}^{0}(t), & \mu_{4}(z(t))=F_{1}^{0}(t) F_{2}^{1}(t) F_{3}^{1}(t) \\
\mu_{5}(z(t))=F_{1}^{1}(t) F_{2}^{0}(t) F_{3}^{0}(t), & \mu_{6}(z(t))=F_{1}^{1}(t) F_{2}^{0}(t) F_{3}^{1}(t) \\
\mu_{7}(z(t))=F_{1}^{1}(t) F_{2}^{1}(t) F_{3}^{0}(t), & \mu_{8}(z(t))=F_{1}^{1}(t) F_{2}^{1}(t) F_{3}^{1}(t)
\end{array}
$$

where $z(t)=\left[\begin{array}{lll}z_{1}(t) & z_{2}(t) & z_{3}(t)\end{array}\right]^{T}$. Due the lake of space the matrices of the T-S model are omitted. The figure 2 illustrates the exactness between the nonlinear model and the T-S one.
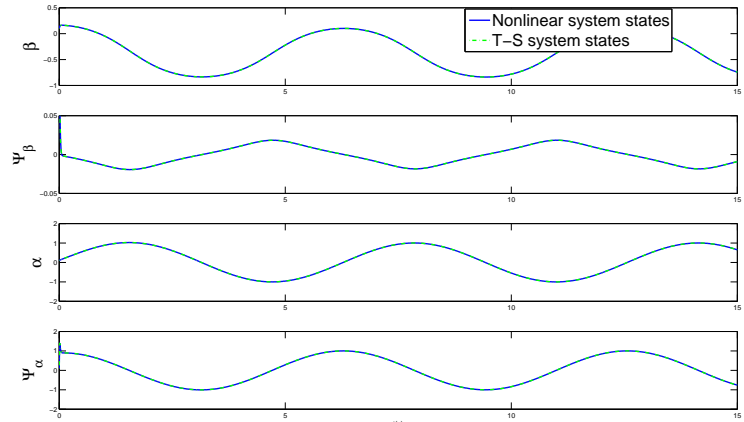

Fig. 2. States of the nonlinear system and those of the T-S system
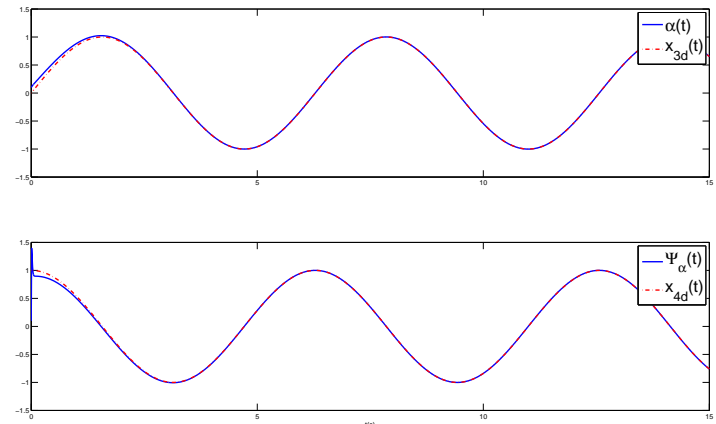

Fig. 3. Trajectory tracking control

\section{OBSERVER DESIGN}

After stabilizing the system, an observer is proposed to estimate the unknown states in the form of equation (6) Assume that only the first and the third components of the state vector are measured, which leads to the output equation

$$
y(t)=C x(t), \quad C=\left(\begin{array}{cccc}
1 & 0 & 0 & 0 \\
0 & 0 & 1 & 0
\end{array}\right)
$$


Note that the state $x_{2}(t)$ is not measured and it appears in the weighting functions of the T-S system, then the weighting functions of the observer must depend on the estimated state $\hat{x}_{2}(t)$. Then the classical approaches cannot be used. The proposed approach is applied and the state estimation error is given in the form

$$
\dot{e}(t)=\sum_{i=1}^{4} \sum_{j=1}^{8} h_{i}(\xi) \mu_{j}(\hat{z})\left(\mathscr{A}_{i}-L_{j} C\right) e(t)
$$

where $\mathscr{A}_{i}$ are obtain from

$$
\frac{\partial f}{\partial x}(\xi)=\sum_{i=1}^{4} \sum_{j=}^{4} e_{n}^{T}(i) e_{n}(j) \frac{\partial f_{i}}{\partial x_{j}}\left(z^{i}\right)
$$

Following the proposed approach, we have firstly

$$
\frac{\partial f}{\partial x}(z)=\left(\begin{array}{cccc}
0 & 30 & 0 & 0 \\
0 & 0 & 0 & 0 \\
0 & 0 & 0 & 1 \\
0 & \frac{\partial f_{4}}{\partial x_{2}}(z) & \frac{\partial f_{4}}{\partial x_{3}}(z) & 0
\end{array}\right)
$$

where

$$
\frac{\partial f_{4}}{\partial x_{2}}(z)=100 \cos \left(z_{3}\right) z_{2} \sin \left(z_{3}\right)+100 \cos \left(z_{3}\right) \sin \left(z_{3}\right)+\frac{1}{3}
$$

$$
\begin{aligned}
\frac{\partial f_{4}}{\partial x_{3}}(z) & =16.35 \cos \left(z_{3}\right)+100 z_{2}^{2} \cos \left(z_{3}\right)^{2}-8.33 u \sin \left(z_{3}\right) \\
& -100 z_{2}^{2} \sin \left(z_{3}\right)^{2}+\frac{1}{3}
\end{aligned}
$$

Defining $\xi_{1}=\frac{\partial f_{4}}{\partial x_{2}}(z)$ and $\xi_{2}=\frac{\partial f_{4}}{\partial x_{3}}(z)$ as new premise variables it is easy to compute a T-S representation of the jacobian $\frac{\partial f}{\partial x}(z)$ in the form

$$
\frac{\partial f}{\partial x}(z)=\sum_{i=1}^{4} \sum_{j=}^{4} e_{n}^{T}(i) e_{n}(j) \frac{\partial f_{i}}{\partial x_{j}}\left(z^{i}\right)=\sum_{i=1}^{4} h_{i}(x) \mathscr{A}_{i}
$$

The premise variables satisfy $84.92 \leq \xi_{1} \leq 167.83$ and $8.9 \leq$ $\xi_{2} \leq 19.95$, which leads to

$$
\begin{aligned}
& \tilde{F}_{1}^{0}=\frac{\xi_{1}-84.92}{82.91}, \tilde{F}_{1}^{1}=\frac{167.83-\xi_{1}}{82.91} \\
& \tilde{F}_{2}^{0}=\frac{\xi_{2}-8.9}{11.05}, \quad \tilde{F}_{2}^{1}=\frac{19.95-\xi_{2}}{11.05}
\end{aligned}
$$

and finally the weighting functions are given by

$$
\begin{aligned}
& h_{1}(z)=\tilde{F}_{1}^{1} \tilde{F}_{2}^{1}, h_{2}(z)=\tilde{F}_{1}^{0} \tilde{F}_{2}^{1} \\
& h_{3}(z)=\tilde{F}_{1}{ }^{1} \tilde{F}_{2}^{0}, h_{4}(z)=\tilde{F}_{1}^{0} \tilde{F}_{2}^{0}
\end{aligned}
$$

with the matrices

$$
\begin{gathered}
\mathscr{A}_{1}=\left(\begin{array}{cccc}
0 & 30 & 0 & 0 \\
0 & 0 & 0 & 0 \\
0 & 0 & 0 & 1 \\
0 & 84.92 & 8.9 & 0
\end{array}\right), \mathscr{A}_{2}=\left(\begin{array}{cccc}
0 & 30 & 0 & 0 \\
0 & 0 & 0 & 0 \\
0 & 0 & 0 & 1 \\
0 & 167.83 & 8.9 & 0
\end{array}\right), \\
\mathscr{A}_{3}=\left(\begin{array}{cccc}
0 & 30 & 0 & 0 \\
0 & 0 & 0 & 0 \\
0 & 0 & 0 & 1 \\
0 & 84.92 & 19.95 & 0
\end{array}\right), \mathscr{A}_{4}=\left(\begin{array}{cccc}
0 & 30 & 0 & 0 \\
0 & 0 & 0 & 0 \\
0 & 0 & 0 & 1 \\
0 & 167.83 & 19.95 & 0
\end{array}\right)
\end{gathered}
$$

The stability of the system (29) is guaranteed if a solution exists to the LMI constraints (23). And in the same time
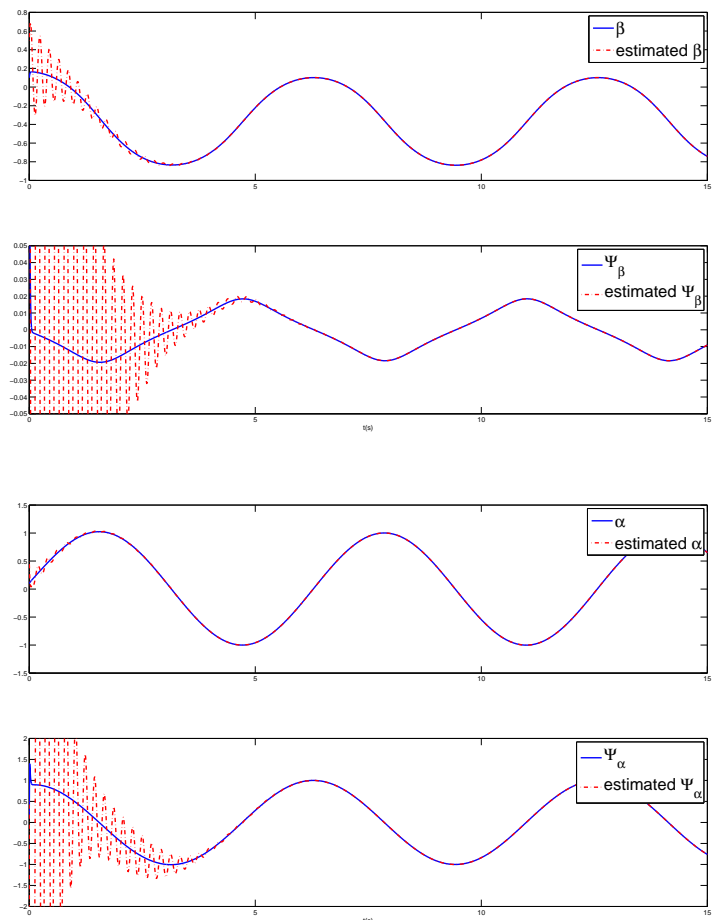

Fig. 4. State estimation without enhanced performances

the gains $L_{j}, \quad j=1, \ldots, 8$ of the observer are obtained by $L_{j}=P^{-1} K_{j}$. The simulation results are given in the figure 4. We can point out that the observer dynamics present an oscillaory phenomenon. The performances of the observer can be improved by pole assignment in an LMI region. we propose an extension of the previous method of synthesis by placing the eigenvalues of the observer in LMI region $S$ defined by $S=\{z \in C|\operatorname{Re}(z)<-a| z \mid,<R\}$, which is an intersection between the left plan defined by the $\operatorname{Re}(z)<$ $-a$ and the disc with center $(0,0)$ and radius $R$. So, the problem is now to solve simultaneously the LMIs (23) and the LMI constraints, corresponding to the LMI region, given as follows (for more details see [7])

$$
\begin{gathered}
A_{i}^{T} P+P A_{i}-K_{j} C-C^{T} K_{j}^{T}+2 a P<0 \\
{\left[\begin{array}{cc}
-R P & P A_{i}-K_{j} C \\
A_{i}^{T} P-C^{T} K_{j}^{T} & -R P
\end{array}\right]<0} \\
i=1, \ldots, 4, \quad j=1, \ldots, 8
\end{gathered}
$$

With adequate choice of $a$ and $R$, these new conditions provides gains $L_{j}$ that reduce the imaginary part of the poles of the dynamics of the state estimation error which ensure the oscillatory phenomenon avoidance and enhances the transient phase of the observer as shown in the figure 5 .

\section{CONCLUSIONS AND FUTURE WORKS}

In this work, an observer methodology is proposed for a nonlinear autonomous bycicle system. The approach is based on T-S fuzzy modeling and differential mean value theorem (DMVT). First, the nonlinear model of the system is 

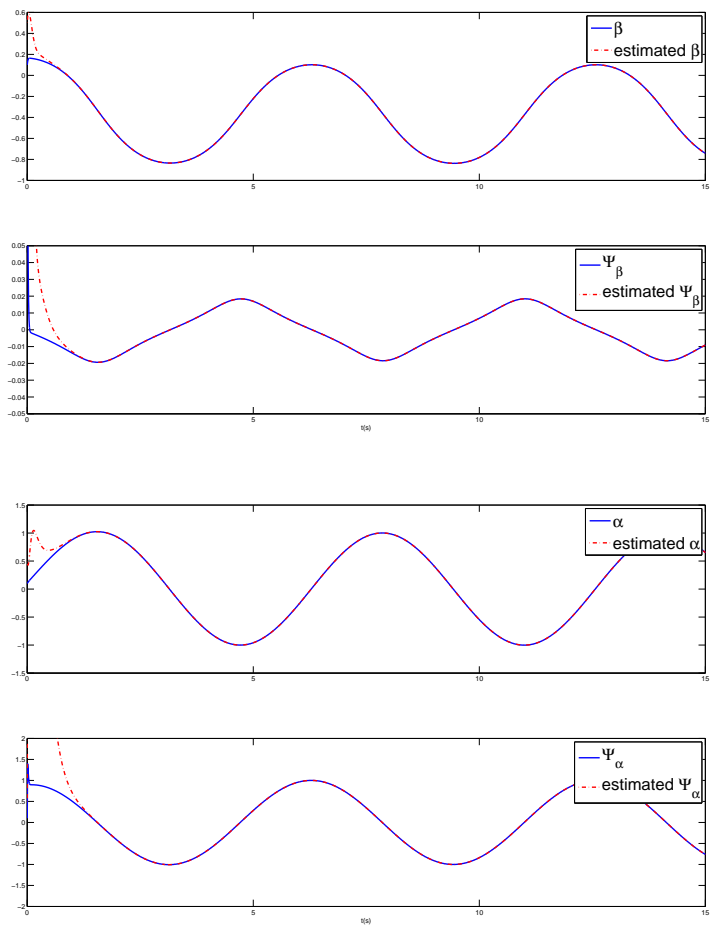

Fig. 5. States and their estimations

transformed to a T-S fuzzy representation with unmeasurable premise variables due to their dependence on unmeasured states. The obtained model is derived form the sector nonlinearity approach which provides an exact T-S model with no loss of information. Secondly, an observer for this type of systems is proposed. The state estimation error is computed and made on the form of autonomous T-S model with the differential mean value theorem. The new structure of the state estimation error allows to use the Lyapunov stability analysis. Then, stability conditions are obtained and formalized in linear matrix inequality (LMI) constraints. An application on bicycle system is proposed to illustrate the proposed approach. The aim of this paper is the observer design for safety, so, since the system is unstable a classic tracking control law is applied to stabilize it and allows to track a reference trajectory. After, an observer is designed for the controlled system, as future works, it is interesting to develop an observer- based control law with T-S approach by using this observer.

\section{REFERENCES}

[1] A. Akhenak, M. Chadli, J. Ragot, and D. Maquin. Design of sliding mode unknown input observer for uncertain Takagi-Sugeno model. In 15th Mediterranean Conference on Control and Automation, MED'07, Athens, Greece, 2007.

[2] A. Akhenak, M. Chadli, J. Ragot, and D. Maquin. Fault detection and isolation using sliding mode observer for uncertain Takagi-Sugeno fuzzy model. In 16th Mediterranean Conference on Control and Automation Congress Centre, Ajaccio, France, June 25-27 2008.

[3] G. I. Bara, J. Daafouz, F. Kratz, and J. Ragot. Parameter dependent state observer design for affine LPV systems. International Journal of Control, 74(16):1601-1611, November 2001.
[4] P. Bergsten, R. Palm, and D. Driankov. Fuzzy observers. In IEEE International Fuzzy Systems Conference, Melbourne, Australia, 2001.

[5] P. Bergsten, R. Palm, and D. Driankov. Observers for Takagi-Sugeno fuzzy systems. IEEE Transactions on Systems, Man, and Cybernetics - Part B: Cybernetics, 32(1):114-121, 2002.

[6] M. Chadli, D. Maquin, and J. Ragot. Non quadratic stability analysis of Takagi-Sugeno systems. In IEEE Conference on Decision and Control, CDC'2002, Las Vegas, Nevada, USA, 2002.

[7] M. Chilali and P. Gahinet. H-infinity design with pole placement constraints : an LMI approach. IEEE Transactions on Automatic Control, 41(3):358-367, 1996.

[8] M. Darouach, M. Zasadzinski, and S.J. Xu. Full-order observers for linear systems with unknown inputs. IEEE Transactions on Automatic Control, 39(3):606-609, March 1994.

[9] Y. Fenge. Stabilizing control design of a motorcycle. Master's thesis, RMIT University, 2007.

[10] N. Getz and J. Marsden. Control of an autonomous bicycle. In IEEE International Conference on Robotics and Automation, pages 13971420, 1995.

[11] T-M. Guerra, A. Kruszewski, L. Vermeiren, and H. Tirmant. Conditions of output stabilization for nonlinear models in the TakagiSugeno's form. Fuzzy Sets and Systems, 157(9):1248-1259, May 2006.

[12] A. Kruszewski, R. Wang, and T.M. Guerra. Nonquadratic stabilization conditions for a class of uncertain nonlinear discrete time TS fuzzy models: A new approach. IEEE Transactions on Automatic Control, 53(2):606 - 611, March 2008.

[13] A. Kruzewski, R. Wang, and T.M. Guerra. Non-quadratic stabilization conditions for a class of uncertain non linear discrete-time T-S fuzzy models: a new approach. IEEE Transactions on Automatic Control, 53(2):606-611, 2008

[14] D.G. Luenberger. An introduction to observers. IEEE Transactions on Automatic Control, 16:596-602, 1971.

[15] B. Marx, D. Koenig, and J. Ragot. Design of observers for takagi sugeno descriptor systems with unknown inputs and application to fault diagnosis. IET Control Theory and Application, 1:1487-1495, 2007.

[16] A.M. Nagy, G. Mourot, B. Marx, G. Schutz, and J. Ragot. Model structure simplification of a biological reactor. In 15th IFAC Symposium on System Identification, SYSID'09, Saint Malo, France, 2009.

[17] R. Palm and P. Bergsten. Sliding mode observers for Takagi-Sugeno fuzzy systems. 9th IEEE International Conference on Fuzzy Systems, FUZZ IEEE, San Antonio, TX, USA, 2000.

[18] R.J. Patton, J. Chen, and C.J. Lopez-Toribio. Fuzzy observers for nonlinear dynamic systems fault diagnosis. In 37th IEEE Conference on Decision and Control, Tampa, Florida USA, 1998.

[19] A.M. Pertew, H.J. Marquez, and Q. Zhao. $\mathrm{H}_{\infty}$ observer design for lipschitz nonlinear systems. IEEE Transactions on Automatic Control, 51:1211-1216, 2006.

[20] S. Raghavan and J. K. Hedrick. Observer design for a class of nonlinear systems. International Journal of Control, 59(2):515-528, 1994.

[21] R. Rajamani. Observers for Lipschitz nonlinear systems. IEEE Transactions on Automatic Control, 43:397-401, March 1998.

[22] Antonio Sala and Carlos Ario. Asymptotically necessary and sufficient conditions for stability and performance in fuzzy control: Applications of polya's theorem. Fuzzy Sets and Systems, 158(24):2671-2686, December 2007.

[23] C. Stefano, I. Boniolo, and S. Savaresi. Attitude estimation of a motorcycle via unscented kalman filter. In 5th IFAC Symposium on Mechatronic Systems, Cambridge, Massachusetts, USA., 13-15 September 2010.

[24] T. Takagi and M. Sugeno. Fuzzy identification of systems and its applications to modeling and control. IEEE Transactions on Systems, Man, and Cybernetics, 15:116-132, 1985.

[25] K. Tanaka and H.O. Wang. Fuzzy Control Systems Design and Analysis: A Linear Matrix Inequality Approach. John Wiley and Sons, 2001.

[26] A.P. Teerhuis and S.T.H Jansen. Motorcycle state estimation for lateral dynamics. In Bicycle and Motorcycle Dynamics, Delft, The Netherlands., 2022 October 2010.

[27] F.E. Thau. Observing the state of non-linear dynamic systems. International Journal of Control, 18:471-479, 1973.

[28] J. Yoneyama. $H_{\infty}$ filtering for fuzzy systems with immeasurable premise variables: an uncertain system approach. Fuzzy Sets and Systems, 160(12):1738-1748, 2009. 